\title{
Mechanism of silica-lysozyme composite formation unravelled by in situ fast SAXS
}

\author{
Tomasz M. Stawski ${ }^{* 1}$, Daniela B. van den Heuvel ${ }^{2,3}$, Rogier Besselink ${ }^{1,4}$, \\ Dominique J. Tobler ${ }^{5}$ and Liane G. Benning ${ }^{1,2,6}$
}

\section{Full Research Paper}

\section{Address:}

${ }^{1}$ German Research Centre for Geosciences, GFZ, Interface Geochemistry, Telegrafenberg, 14473, Potsdam, Germany, ${ }^{2}$ School of Earth and Environment, University of Leeds, Woodhouse Lane, LS2 9 JT, Leeds, UK, ${ }^{3}$ Rock-Water Interaction Group, Institute of Geological Sciences, University of Bern, Baltzerstrasse 3, 3012, Bern, Switzerland, ${ }^{4}$ Université Grenoble Alpes, Université Savoie Mont Blanc, CNRS, IRD, IFSTTAR, ISTerre, 38000 Grenoble, France, ${ }^{5}$ Nano-Science Center, Department of Chemistry, University of Copenhagen, Universitetsparken 5, 2100, Copenhagen, Denmark and ${ }^{6}$ Department of Earth Sciences, Free University of Berlin, Malteserstr. 74-100 / Building A, 12249, Berlin, Germany

\section{Email:}

Tomasz M. Stawski* - stawski@gfz-potsdam.de

* Corresponding author

Keywords:

composite; lysozyme; scattering; silica; small-angle X-ray scattering (SAXS)
Beilstein J. Nanotechnol. 2019, 10, 182-197. doi:10.3762/bjnano.10.17

Received: 09 August 2018

Accepted: 11 December 2018

Published: 14 January 2019

This article is part of the thematic issue "Advanced hybrid nanomaterials".

Guest Editor: V. de Zea Bermudez

(C) 2019 Stawski et al.; licensee Beilstein-Institut. License and terms: see end of document.

\footnotetext{
Abstract

A quantitative understanding of aggregation mechanisms leading to the formation of composites of inorganic nanoparticles (NPs) and proteins in aqueous media is of paramount interest for colloid chemistry. In particular, the interactions between silica $\left(\mathrm{SiO}_{2}\right)$ NPs and lysozyme (LZM) have attracted attention, because LZM is well-known to adsorb strongly to silica NPs, while at the same time preserving its enzymatic activity. The inherent nature of the aggregation processes leading to NP-LZM composites involves structural changes at length scales from few to at least hundreds of nanometres but also time scales much smaller than one second. To unravel these we used in situ synchrotron-based small-angle X-ray scattering (SAXS) and followed the subtle interparticle interactions in solution at a time resolution of $50 \mathrm{~ms} /$ frame $(20 \mathrm{fps})$. We show that if the size of silica NPs (ca. $5 \mathrm{~nm}$ diameter) is matched by the dimensions of LZM, the evolving scattering patterns contain a unique structure-factor contribution originating from the presence of LZM. We developed a scattering model and applied it to analyse this structure function, which allowed us to extract structural information on the deformation of lysozyme molecules during aggregation, as well as to derive the mechanisms of composite formation.
} 


\section{Introduction}

A mechanistic understanding of aggregation in aqueous media leading to the formation of composites of inorganic nanoparticles and proteins is of paramount interest for colloid chemistry, Earth sciences, or the design of protein-sensitized biomedical devices and sensors [1-6]. In a broader perspective, many processes involving the assembly of nanoparticles to higher-level hierarchical structures are known to be directed by small organic molecules and macromolecules [7-10]. Such fundamental interactions are the key to understand the crystallisation of biominerals in living organisms (e.g., in bone formation), and to manufacture better functional materials [11-15].

In particular, composites of amorphous silica $\left(\mathrm{SiO}_{2}\right)$ nanoparticles (NPs) and lysozyme (LZM) have attracted attention because silica NPs readily form in many Earth surface environments (e.g., oceans, hot springs) where biological activity dominates (e.g., diatom formation) but they are also key components in numerous technological applications from electronics to paint production. In turn, lysozyme adsorbs strongly to silica NPs [16-18], while at the same time preserving its enzymatic activity, most notably antibacterial properties [19]. Over a wide range of $\mathrm{pH}$ values (2 to ca. 10), the surface of silica NPs is negatively charged, whereas the LZM molecule is positively charged $[20,21]$. This way LZM can act as a bridge between silica NPs, leading to aggregation and flocculation and thus to large silica NP-LZM composites. In the $\mathrm{SiO}_{2}-\mathrm{LZM}$ model system, a number of studies investigating the relationship between silica NP sizes, and adsorption modes of lysozyme revealed a correlation between composite properties and the folding/structure of the protein, its enzymatic activity and the exact protein localization with respect to the silica NPs inside flocculated composites $[16,17,19,21-24]$. The inherent nature of the aggregation processes leading to silica-lysozyme composites, involves structural changes at length scales from a few to hundreds of nanometres, which makes this system well-suited to be characterized by scattering methods. In particular, recent advances $[25,26]$ in detector technology for synchrotron-based in situ and time-resolved small-angle X-ray scattering (SAXS), now allow one to follow all steps in the formation of $\mathrm{SiO}_{2}-\mathrm{LZM}$ composites from the individual components. Nevertheless, although such scattering data will reflect the in situ state of a system during measurement, the quantitative information related to any changes in structural properties of the particles/species of interest can only be accessed by developing, testing and validating relevant models and bespoke data analysis methods.

In this study we show step-by-step how a scattering model was developed, verified and applied to time-resolved synchrotronbased SAXS data in which we followed in situ the lysozyme-induced aggregation of silica NPs (ca. $5 \mathrm{~nm}$ in diameter) at a time resolution of $50 \mathrm{~ms}$. It is important to note that at typical NPs sizes and concentrations [17,24] and protein concentrations [27] usually employed to make such $\mathrm{SiO}_{2}-\mathrm{LZM}$ composites, the relative X-ray scattering contrast/intensity of lysozyme, in the presence of silica, is insufficient to determine the protein contribution to the overall scattering pattern (ratio of ca. 1:100 between LZM and NPs), and only the NP component can directly be followed. However, through this study we demonstrate that if the size of the silica NPs is matched with the dimensions of lysozyme (ellipsoidal molecule [28] $3 \times 3 \times 4.5 \mathrm{~nm}^{3}$ ), the evolving scattering patterns contain a unique structure-factor contribution originating from the presence of lysozyme, and this way this important contribution can be assessed. The analysis of this structure function through the derived model then allowed us to extract detailed structural information on the deformation of the LZM molecules upon aggregation, and to determine the mechanisms of the formation of $\mathrm{SiO}_{2}-\mathrm{LZM}$ composites.

\section{Results and Discussion}

This section of the manuscript is organised in the following way: (1) data presentation and a discussion of general trends; (2) derivation of a self-consistent mathematical model to fit the timer-resolved scattering curves; (3) presentation of the quantitative results from the model fits; (4) discussion of the quantitative trends.

\section{Evolution of SAXS patterns and derived aggregation stages}

Upon mixing of the silica NPs and the LZM solution we observed very fast flocculation, which indicated the formation of the composites. In Figure 1 we show an overview of these formation processes based on SAXS data collected at a time resolution of $50 \mathrm{~ms}$ and spanning ca. $300 \mathrm{~s}$.

In a contour plot of the time-resolved scattering patterns (Figure 1A), one can distinguish four characteristic time periods (I-IV) and one region of interest (ROI V), which spanned through periods II to IV. Period I corresponds to the initial ca. $24 \mathrm{~s}$ of the scattering patterns of silica NPs before the injection of lysozyme. Based on this data we determined the initial form factor (size distribution) of the silica NPs prior to mixing with lysozyme (Figure 1B). The scattering pattern in a log-log representation prominently flattens out at low $q$ (i.e., $I(q) \propto q^{0}$ ). This shows that the initial silica NPs were not aggregated and well-suspended. We derived a discrete size distribution (histogram in the inset of Figure 1B) for the NPs from the Monte Carlo fit implemented [29,30] in MCSAS under the a priori assumption that the NPs were spherical in shape [31] (physicochemical parameters of amorphous silica given in Table S1, Supporting Information File 1). The as-obtained histogram indi- 


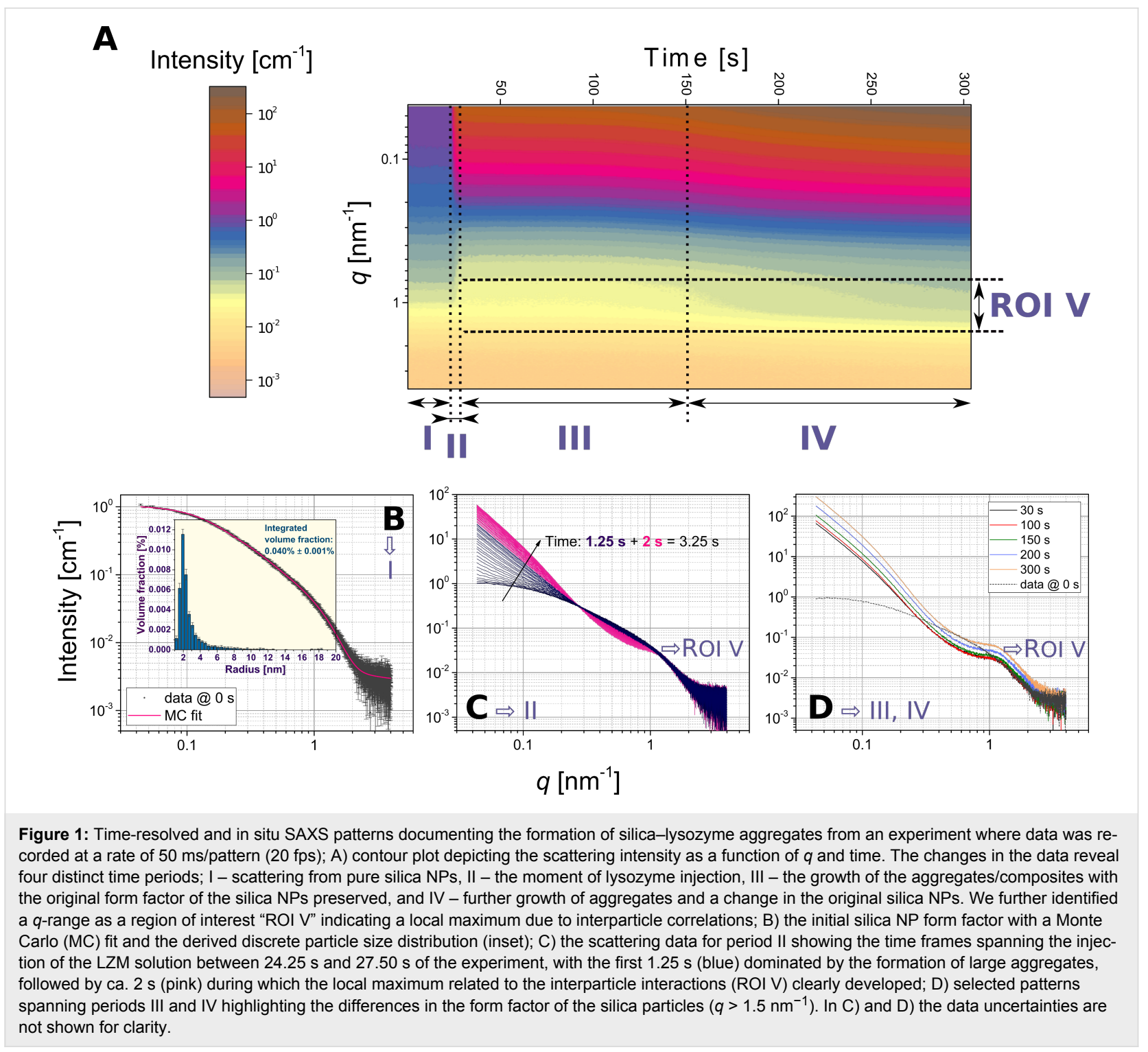

cated that the size distribution was relatively narrow with a mean radius of $2.53 \pm 0.01 \mathrm{~nm}$ (distribution statistics given in Table S2, Supporting Information File 1). The total integrated volume fraction for the NPs obtained from the fit, was $0.040 \%$ $\pm 0.001 \%$, which matches very closely the expected value of $0.041 \%$ calculated for silica NP precipitated from a $1000 \mathrm{ppm}$ $\mathrm{SiO}_{2}$ solution at $\mathrm{pH} 7.5$ and $21{ }^{\circ} \mathrm{C}$ (Table S1, Supporting Information File 1, calculated with PHREEQC [32]).

Period II (ca. 25-30 s) in Figure 1A represents scattering patterns during and soon after the injection of the LZM solution and its mixing with the silica NPs. Period II is hence preceded by a dead-time period of $500 \mathrm{~ms}$ (see Experimental section). Period II (Figure 1C) can be divided into multiple steps. The first $1.25 \mathrm{~s}$ were primarily characterized by a rapid and significant (ca. 11-fold) increase in intensity at low $q$ $\left(q<0.3 \mathrm{~nm}^{-1}\right)$. During the following $2 \mathrm{~s}$, the low- $q$ part still kept increasing (to ca. 15-times of the initial intensity) but less rapidly, and at $q \approx 1 \mathrm{~nm}^{-1}$ a characteristic local maximum developed (ROI V in Figure 1A). The intensity increase at low $q$ originated from the formation of large aggregates constituting the composites, with sizes outside the minimum $q$-range, whereas the local maximum (the correlation peak $q \approx 1 \mathrm{~nm}^{-1}$ ) indicated the presence of interparticle correlations within those aggregates.

The intensity increase at low $q$ associated with the aggregation continued throughout period III (between ca. 30 and $150 \mathrm{~s}$ ), yet the correlation peak in ROI V did not change significantly (Figure 1D). Note that up to $150 \mathrm{~s}$ (periods I-III) the high- $q$ part of the data $\left(q>1.5 \mathrm{~nm}^{-1}\right.$, Figure 1C,D) did not change, indicating that the original form factor of silica NPs remained the same 
after the injection of lysozyme. Therefore, as a first approximation the observed electron-density scattering contrast in these periods (I-III) can be interpreted to originate solely from the silica NPs and not from the combination of silica and lysozyme (Figure S1, Supporting Information File 1). Hence, we could treat the system as a system with two different electron densities (silica NPs and solvent matrix). However, because our silica nanoparticles were smaller (ca. $5 \mathrm{~nm}$ ) compared to silica NPs in previous scattering studies (ca. $20 \mathrm{~nm}$ ) on silica-protein composite formation $[16,17,23,24]$ and because the lysozyme addition dramatically changed the silica aggregation state, the contributions of the lysozyme scattering can be accounted for indirectly from the interparticle correlations observed in ROI V. This is key here, because it allows us to extract the changes in lysozyme structural properties as the composites evolve over time, without deriving circumstantial models for a system with three electron densities (silica, lysozyme, and the solvent matrix).

In period IV (150-300 s, Figure 1A and Figure 1D), we observed a further intensity increase at low $q$ (three-times higher at $300 \mathrm{~s}$ than at $150 \mathrm{~s}$ ), which indicated a continuous increase in aggregate size from periods II and III. In time period IV the intensity of ROI V (Figure 1A) started to increase together with the silica form factor at high $q\left(q>1.5 \mathrm{~nm}^{-1}\right.$, Figure 1D). This suggests that as aggregation continued between 150 and $300 \mathrm{~s}$, the silica NPs themselves started to grow, e.g., due the coalescence of NPs or similar processes (under an assumption that the particles remained spherical in shape).

\section{Scattering model}

In the considered silica-LZM composites, the scattering contrast originating from the lysozyme itself can be mostly disregarded (see Figure S1, Supporting Information File 1). Hence, the scattering contrast of the primary silica particles, $\left(\Delta \rho_{\mathrm{p}}\right)^{2}$, - expressed as the difference in scattering length density, SLD - is equal to the squared difference in the SLDs of silica and the surrounding water matrix (Table S1, Supporting Information File 1). Consequently, using such an approximation allows us to deal with a two-electron-density system, where the scattering intensity, $I(q)$ is a product of the scattering contrast, $\left(\Delta \rho_{\mathrm{p}}\right)^{2}$, the form factor of the silica NPs, $P_{\mathrm{p}}(q)$, weighted by a volume fraction of silica NPs, $\phi_{\mathrm{p}}$, and their volume, $V_{\mathrm{p}}$, together with an effective structure-factor function describing the spatial arrangement of silica NPs within the aggregates, $S_{\text {eff }}(q)$ (Equation 1). We use subscript "p" to emphasize that the parameters and functions concern the primary silica NPs.

$$
I(q)=\left(\Delta \rho_{\mathrm{p}}\right)^{2} \cdot \phi_{\mathrm{p}} \cdot V_{\mathrm{p}} \cdot P_{\mathrm{p}}(q) \cdot S_{\text {eff, } \mathrm{p}}(q) .
$$

Equation 1 is valid only for a system of ideally monodisperse particles (i.e., the distribution is a delta function), which is actually not the case for the silica NPs used here (Figure 1B). This is an important consideration when including the interparticle interactions from the structure factor. There are several approaches to consider the polydispersity of particles together with a structure factor [33], but because the fitted size distribution (histogram in Figure 1B) is discrete with a finite number of $n$ bins a local monodisperse approximation (LMA) [34] is used in our models:

$$
I(q)=\left(\Delta \rho_{\mathrm{p}}\right)^{2} \sum_{i=1}^{n}\left(\phi_{\mathrm{p}, i}\left(r_{i}\right) \cdot V_{\mathrm{p}}\left(r_{i}\right) \cdot P_{\mathrm{p}}\left(q, r_{i}\right) \cdot S_{\mathrm{eff}, \mathrm{p}}\left(q, r_{i}\right)\right),
$$

where $P_{\mathrm{p}}\left(q, r_{i}\right)$ is the form factor of a sphere of radius $r_{i}$.

The discrete size distribution (Figure 1B) has the form of Equation 3, where for each size contribution $\left(r_{i}\right)$ the corresponding volume fractions, $\phi_{i}$, are known:

$$
D\left(r_{i}\right)=\sum_{i=1}^{n} \phi_{\mathrm{p}, i}\left(r_{i}\right)
$$

Since the partial $i$-th structure factor, $S_{\text {eff }}\left(q, r_{i}\right)$, includes the interparticle correlations between silica NPs and lysozyme (the local maximum at $q_{\mathrm{max}} \approx 1 \mathrm{~nm}^{-1}$, ROI V in Figure 1), it is also dependent on $r_{i}$ of the primary silica NPs. Additionally, the structure factor expression has to account for the aggregation of the silica NPs to large objects (the low- $q$ increase), yet the size of these aggregates, in turn, does not necessarily depend on the size of the primary silica NPs.

\section{The interparticle correlations and the local maximum}

Under the considered physicochemical conditions the inorganic silica NPs and the protein molecules are oppositely charged [21], and hence they interact through the attractive potential. This, in turn, leads to the formation of aggregates/composites in which NPs behave closely to adhesive hard spheres. The aforementioned interactions and the arrangement of particles in space lead to the occurrence of a broad maximum in the scattering pattern (Figure 1, ROI V). We simulate these effects by implementing the adhesive hard-sphere structure factor, $S_{\mathrm{SHS}}(q)[35-$ 37]. The interaction between particles at the distance $x$ is approximated by the following potential $U(x)$ :

$$
\frac{U(x)}{k_{\mathrm{B}} T}=\left\{\begin{array}{c}
\infty \Rightarrow 0<x<2 R_{\mathrm{HS}} \\
\ln \left[12 \tau \Delta /\left(2 R_{\mathrm{HS}}+\Delta\right)\right] \Rightarrow 2 R_{\mathrm{HS}}<x<2 R_{\mathrm{HS}}+\Delta,(4) \\
0 \Rightarrow x>2 R_{\mathrm{HS}}+\Delta
\end{array}\right.
$$


where $R_{\mathrm{HS}}$ is a hard sphere radius of particles, $\Delta$ is the width of a potential well, and $\tau$ is a stickiness parameter. The structure factor expression, $S_{\mathrm{SHS}}(q)$, is defined through the following set of equations (Equations 5-14). The structure factor is a function of four parameters in Equation 5, whereas the remaining variables and the associated expressions in Equations 6-14 are merely auxiliary by convention, so that we avoid writing a very long single expression. They should be consequently substituted into each other where suitable to obtain the final expression.

$$
\begin{aligned}
& S_{\mathrm{SHS}}\left(q, R_{\mathrm{HS}}, v, \tau\right)=\frac{1}{1-C(q)} \\
& \kappa=2 q R_{\mathrm{HS}} \\
& \eta=v\left(\frac{2 R_{\mathrm{HS}}+\Delta}{2 R_{\mathrm{HS}}}\right)^{3} \\
& \varepsilon=\tau+\frac{\eta}{1-\eta} \\
& \gamma=v \frac{1+\eta / 2}{3(1-\eta)^{2}} \\
& \lambda=\frac{6}{\eta}\left(\varepsilon-\sqrt{\varepsilon^{2}-\gamma}\right) \\
& \mu=\lambda \eta(1-\eta) \\
& \alpha=\frac{(1+2 \eta-\mu)^{2}}{(1-\eta)^{4}} \\
& \beta=-\frac{3 \eta(2+\eta)^{2}-2 \mu\left(1+7 \eta+\eta^{2}\right)+\mu^{2}(2+\eta)}{2(1-\eta)^{4}}
\end{aligned}
$$

In this study, the stickiness $\tau$ is calculated from Equation 4, based on the literature data for silica-LZM composites synthesized under similar physicochemical conditions [24]: $U\left(2 R_{\mathrm{HS}}<x<2 R_{\mathrm{HS}}+\Delta\right)=-2.5 k_{\mathrm{B}} T ; \Delta=0.1 \cdot\left(2 R_{\mathrm{HS}}\right)$ under the restriction that $\Delta_{\min } \geq 0.15 \mathrm{~nm}$ (i.e., the average H-bond length). The value of $U$ in our study may slightly differ from literature values, because for constant $\mathrm{pH}$ value and salinity, the surface charge of NPs increases with decreasing size [38]. However, it is unlikely that $U>-3 k_{\mathrm{B}} T$, and within the considered range, the value of $U$ will not affect our fitting results. $v$ is a local packing parameter, i.e., a local volume fraction within the aggregate, and for the random packing of polydisperse spheres it does not exceed 0.65 [39-42]. Figure 2 shows the scattering patterns at 0 and $100 \mathrm{~s}$ (Figure 1D) together with simulated curves based on Equation 2, in which the contributions of the silica NPs were taken from the Monte Carlo-fitted form factor (Figure 1B), while the contribution of $S_{\text {eff }}\left(q, r_{i}\right)$ was included from Equation 5 (and Equations 6-14).

The simulations show the important effects that polydispersity has on the structure factor and the position of the correlation peak in ROI V. Typically, for correlations originating from (sticky) hard-sphere interactions, one considers the following dependence, for the approximated position of the peak at $q_{\text {max }}$ :

$$
2 R_{\mathrm{HS}} \approx \frac{2 \pi}{q_{\max }} .
$$

Equation 15 infers that the expected average hard-sphere radius, $R_{\mathrm{HS}}$, would be equal to the mean radius of a silica particle $<r>\approx 2.5 \mathrm{~nm}$ (Figure 1B, Table S2, Supporting Information File 1). This, in turn, suggests that silica NPs on average touch each other without any LZM molecules in between, or that the protein molecules, if present within the aggregates and among individual silica NPs, are very strongly deformed, likely to a point that they barely contribute to the determined $R_{\mathrm{HS}}$. Nevertheless, the simulation in Figure 2A clearly shows that if the size distribution of silica NPs is actually correctly accounted for, then in order to fit the peak position accurately, an additional "spacer", $a R_{\mathrm{eHS}}$ (additional effective hard sphere radius) has to be included in Equation 2 and Equations 5-14:

$$
R_{\mathrm{HS}, i}=r_{i}+a R_{\mathrm{eHS}} .
$$

$$
\begin{aligned}
C(q) & =2 \frac{\eta \lambda}{\kappa} \sin \kappa-2 \frac{\eta^{2} \lambda^{2}}{\kappa^{2}}(1-\cos \kappa) \\
& -\left[\alpha \kappa^{3}(\sin \kappa-\kappa \cos \kappa)+\beta \kappa^{2}\left(2 \kappa \sin \kappa-\left(\kappa^{2}-2\right) \cos \kappa-2\right)\right. \\
& \left.+\frac{\eta \alpha}{2}\left(\left(4 \kappa^{3}-24 \kappa\right) \sin \kappa-\left(\kappa^{4}-12 \kappa^{2}+24\right) \cos \kappa+24\right)\right] \cdot 24 \frac{\eta}{\kappa^{6}}
\end{aligned}
$$




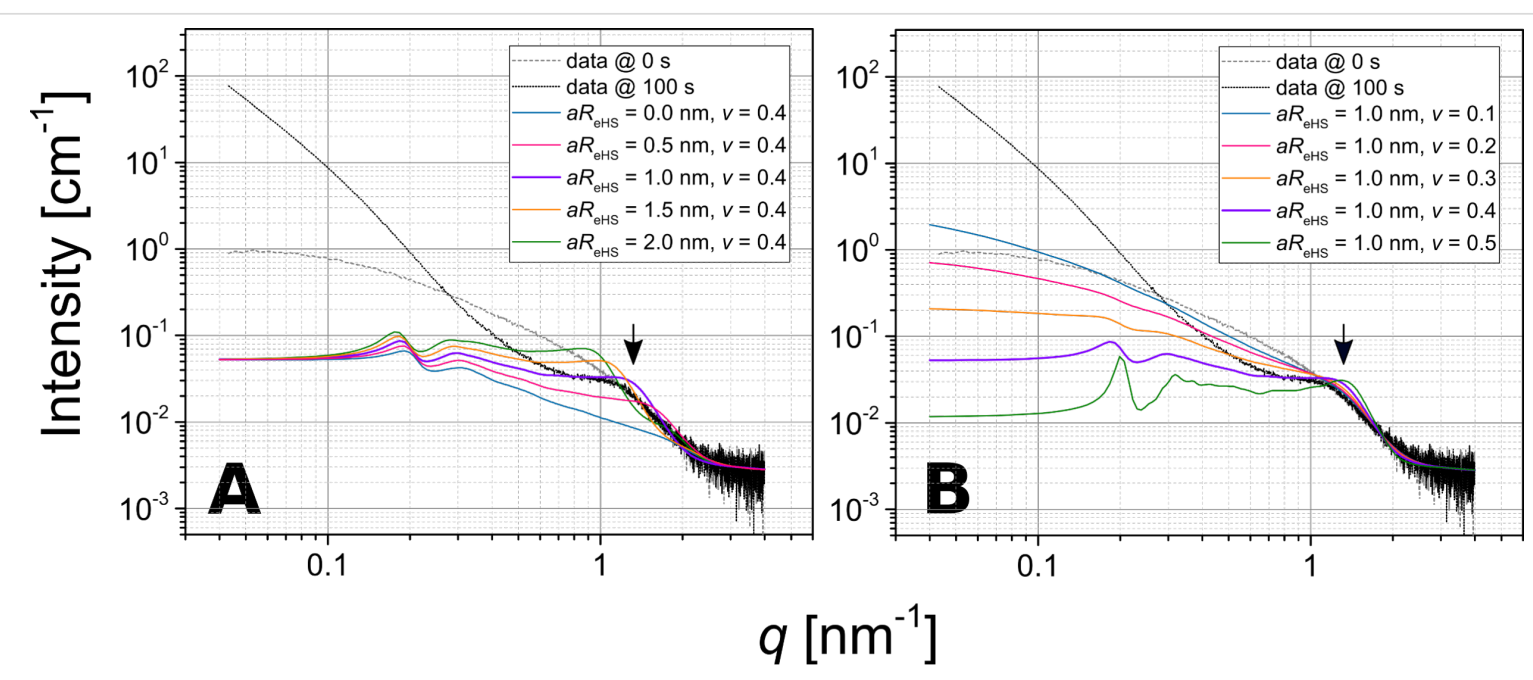

Figure 2: Comparison of the measured (black) and simulated (coloured) scattering patterns to illustrate the contribution of $S_{S H S}(q)$ from Equations $5-14$, plugged into Equation 2 as the only structure-factor expression. It was the aim to represent the correlation peak at $q \approx 1 \mathrm{~nm}^{-1}$, whereas the effects at low $q$ are further discussed in the text and in Figure 3. A) The position of the correlation peak at $q \approx 1 \mathrm{~nm}^{-1}$ (arrow) is reproduced more accurately by $S_{\mathrm{SHS}}(q)$ for the polydisperse silica NPs (Figure 1B), only if one considers an additional effective hard sphere radius, $a R_{\mathrm{eHS}}$

(Equation 16); B) the effect of the local volume fraction $v$ onto the intensity of the correlation peak (arrow). The data uncertainties are not shown for clarity.

By setting merely $r_{i}=R_{\mathrm{HS}, i}$ (i.e., $a R_{\mathrm{eHS}}=0$ ) the position of the simulated peak visibly shifts towards higher $q$ values with respect to the measured peak. Here, $a R_{\mathrm{eHS}}$ is associated with the presence of a single LZM molecule located in between individual silica NPs with the diameter of the LZM molecule represented by $2 a R_{\mathrm{eHS}}$. The simulation in Figure $2 \mathrm{~B}$ also shows that the packing factor $v$ within the aggregates, which directly correlates with the intensity of the broad peak around $q \approx 1 \mathrm{~nm}^{-1}$, has to be relatively high ( $v$ above ca. 0.4 ) in order to be able to simulate the intensity profile at $q \approx 1 \mathrm{~nm}^{-1}$ in the later stages (ca. $100 \mathrm{~s})$.

\section{Low-q intensity increase, aggregation, and the structure factor expression of aggregates with internal correlations}

The structure factor from Equations 5-14 does not reproduce the observed intensity increase at low $q$ (Figure 1 and Figure 2A), because the sticky hard-sphere structure factor is derived under the assumption that the interactions extend to an infinite length scale, with respect to the probed volume. However, in our experiments, we initially have a finite number of "loose" silica NPs that are then rearranged to large aggregates upon mixing with lysozyme. Hence, microscopically such aggregates must have a finite size, even if their size (radii of gyration) cannot be determined directly from our scattering data, due to the used $q$ range (see Experimental section). However, ultimately, to obtain a good fit an expression for $S_{\text {eff }}(q)$ (Equation 2) has to account for both the fine structure within the aggregates causing the interparticle correlations (as in Equations 5-14) and also the low- $q$ intensity increase due to the presence of the interface between the aggregates/composites and the solvent matrix.

A general expression for such an effective structure factor for particles within an aggregate/droplet was proposed originally by Hashimoto and co-workers [43]. Several variations and applications of this concept are furthermore known [37,44]. For the purpose of the analysis of our data, we further extended the expressions originating from Hashimoto et al. as we show below in a final form. In the Appendix section the complete derivation and the rationale are presented. Our derivation is essential here, because it allowed us to quantify indirectly the changes in the size of the aggregates, although the direct measurement of their radii of gyration was not possible. The so-derived general expression for $S_{\text {eff }}\left(q, r_{i}\right)$ (Equation 17) is expressed as the sum between the structure function of an aggregate ("template"), $S_{\text {agg }}(q)$, and the structure factor of the internal arrangement of the aggregate, $S_{\text {int }}(q)$, which in our case becomes subsisted by $S_{\mathrm{SHS}}(q)$ (Equations 5-14):

$$
\begin{aligned}
S_{\mathrm{eff}}\left(q, r_{i}\right) & =S_{\mathrm{int}}(q)+S_{\mathrm{agg}}(q) \\
& =S_{\mathrm{SHS}}\left(q, R_{\mathrm{HS}, i}, \nu, \tau\right)+A \cdot q^{-D},
\end{aligned}
$$

where $D$ is a fractal dimension describing the arrangement of primary particles within the composites, and $A$ is a single collective fitting parameter in our model, which is proportional to the number density of aggregates, $N_{\text {agg }}$, and their specific surface area, $\mathrm{SSA}_{\mathrm{agg}}$. Hence, it expresses indirectly the size/ 


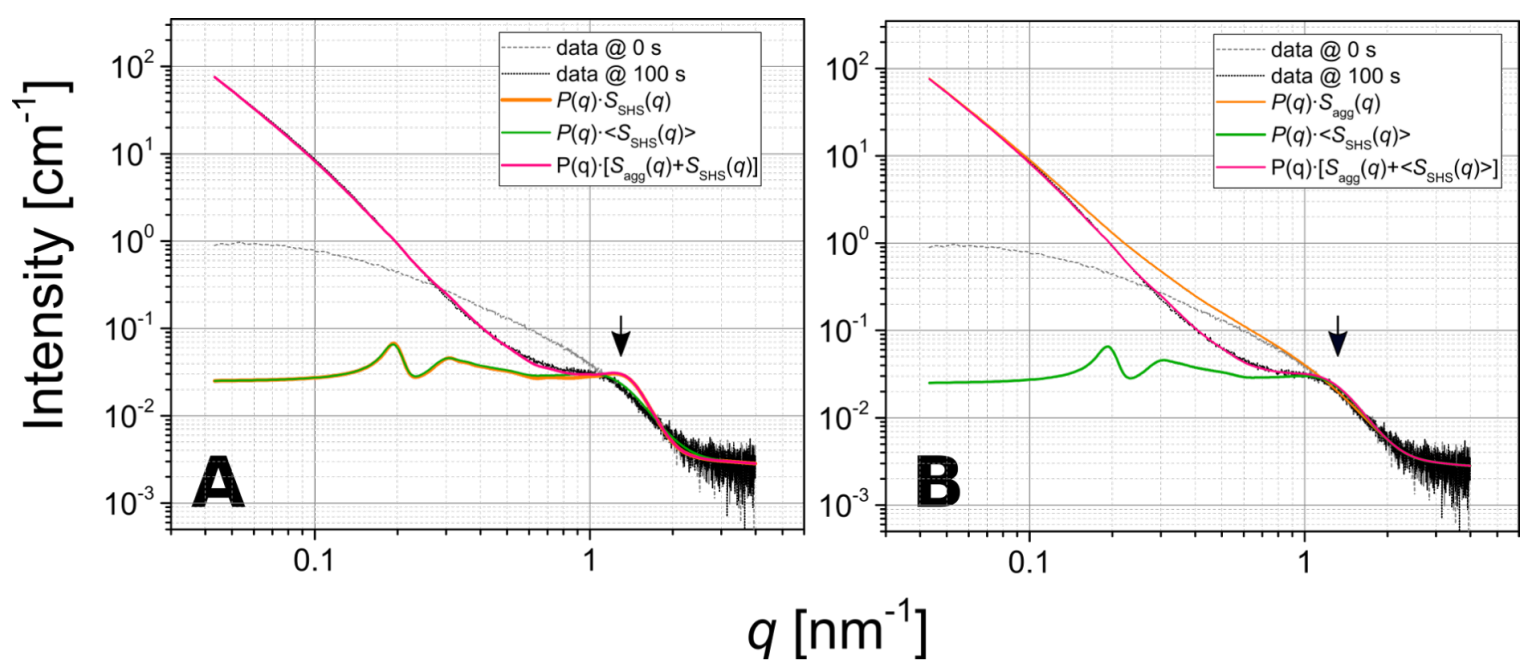

Figure 3: Fitting of the structure-factor contributions to a scattering curve measured at $100 \mathrm{~s}$ during the composite-formation processes. A) Comparison between the effect of the unsmeared (monodisperse $a R_{\mathrm{eHS}}$, red line) $S_{\mathrm{SHS}}(q)$ from Equations 5-14 and the smeared (polydisperse, green line) $<S_{\mathrm{SHS}}(q)>$ from Equation 18. Fits yielded $v=0.454 \pm 0.008, a R_{\mathrm{eHS}}=0.979 \pm 0.036 \mathrm{~nm},<a R_{\mathrm{eHS}}>=0.930 \pm 0.000 \mathrm{~nm}$, and $\sigma=0.533 \pm 0.050 \mathrm{~nm}$; the complete structure factor fit (pink line), which includes the unsmeared $S_{\mathrm{SHS}}(q)$, illustrates that the form of the function affects only the correlation peak at $q \approx 1 \mathrm{~nm}^{-1}$ (arrow), and not the low- $q$ scattering intensity of the aggregates; B) the final fit to the data (pink line) with each structure-factor contribution plotted separately (green and orange lines). Parameters for $\left\langle S_{S H S}(q)>\right.$ are the same as in panel A, whereas for the $S_{\text {agg }}(q)$ from Equation 17 , we obtained $A=0.0437 \pm 0.0001$ and $D=2.37 \pm 0.00$. The data uncertainties are not shown for clarity.

extent of the aggregates. In Figure 3, we show that the fits with the introduced expressions for the partial structure-factor contributions indeed represent the structural features present in the selected scattering pattern (example@100 s).

However, as is evident from Figure $3 \mathrm{~A}$, the $S_{\mathrm{SHS}}(q)$ from Equations 6-14 has to be further improved, because in Figure 3A the correlation peak is relatively broad ("smeared out"), yet still intense. Typically one would expect such a broadened shape if the local volume-fraction parameter, $v$, was smaller than derived from the best fit (i.e., below ca. 0.4). However, this would also inevitably yield a smaller relative intensity of this peak (see Figure 2B). Hence, in order to explain this contradiction, one has to remember that the position of the maximum and its shape are predominantly related to $a R_{\mathrm{eHS}}$. The shape of the peak can be modelled substantially better if one allows for a distribution of this parameter in the fitting routine. The need for such mathematical treatment is in fact a manifestation of the actual physical effects, if we consider that $a R_{\mathrm{eHS}}$ represents a radius of a LZM molecule. A LZM molecule can become, at least partially, heterogeneously deformed (on average, in a global sense) within an aggregate, e.g., due to the variation of local forces, which, in turn, is a consequence of polydispersity of the silica NPs and the random character of the packing of the silica NPs. More importantly, since lysozyme is a small prolate ellipsoidal protein, with its principal semi-axes being $1.5 \mathrm{~nm} \times 1.5 \mathrm{~nm} \times 2.25 \mathrm{~nm}$, the polydispersity in $a R_{\mathrm{eHS}}$ may account for the fact that the protein molecules can be different- ly orientated during adsorption to the NPs. Yet, so far we tried to represent their contribution through a (hard) spherical model. To overcome this, we used a Gaussian distribution to define the average structure factor $\left\langle S_{\mathrm{SHS}, i}(q)>\right.$ in Equation 18. The application of this structure factor ultimately leads to smearing of the maximum at a constant value of $v$ and hence yields significantly improved fits (Figure 3B):

$$
\begin{aligned}
& \left\langle S_{\mathrm{SHS}, i}\left(q, r_{i}+\left\langle a R_{\mathrm{eHS}}\right\rangle, \sigma, v, \tau\right)\right\rangle= \\
& =\frac{\int_{0}^{\left\langle a R_{\mathrm{eHS}}\right\rangle+8 \sigma} D_{\mathrm{G}}\left(\left\langle a R_{\mathrm{eHS}}\right\rangle, \sigma, R\right) \cdot S_{\mathrm{SHS}, i}\left(q, r_{i}+R, \nu, \tau\right) \mathrm{d} R}{\int_{0}^{\left\langle a R_{\mathrm{eHS}}\right\rangle+8 \sigma} D_{\mathrm{G}}\left(\left\langle a R_{\mathrm{eHS}}\right\rangle, \sigma, R\right) \mathrm{d} R}, \\
& D_{\mathrm{G}}\left(\left\langle a R_{\mathrm{eHS}}\right\rangle, \sigma, R\right)=\frac{1}{\sqrt{2 \pi \sigma^{2}}} \exp \left(-\frac{\left(R-\left\langle a R_{\mathrm{eHS}}\right\rangle\right)^{2}}{2 \sigma^{2}}\right) .
\end{aligned}
$$

In Equation 18, the mean of the distribution (Equation 19) is $<a R_{\mathrm{eHS}}>$, whereas $\sigma$ denotes the standard deviation. This is the final expression used to represent $S_{\text {int }}(q)$ in Equation 17 and to fit all the scattering curves from regions II and III in Figure 1. The numerical integration was performed for each $i$-th bin of the discrete size distribution characterizing the form factor (Figure 1B). The complete source code and selected data sets are deposited at GitHub.com [45]. 


\section{Application of the scattering model}

We used the above described scattering model to fit the timeresolved data set and to derive the fitting parameters as a function of the time (Figure 1, Period II, III and beginning of IV, 3010 curves). Figure 4 shows the time dependence of the five model parameters obtained by fitting the scattering data between 24.5 and $175 \mathrm{~s}$.
During the first ca. $5 \mathrm{~s}$ after mixing, the values of $<a R_{\mathrm{eHS}}>$ and $\sigma$ lie way outside reasonable error margins (Figure 4A,B). This is to be expected, because the corresponding local volume fraction, $v$, is very low (well below 0.1 , Figure $4 \mathrm{C}$ ) at the beginning of the composite-formation process (i.e., the beginning of period II in Figure 1). Consequently, the contribution of $<S_{\mathrm{SHS}}(q)>$ to the structure factor during this period is mostly

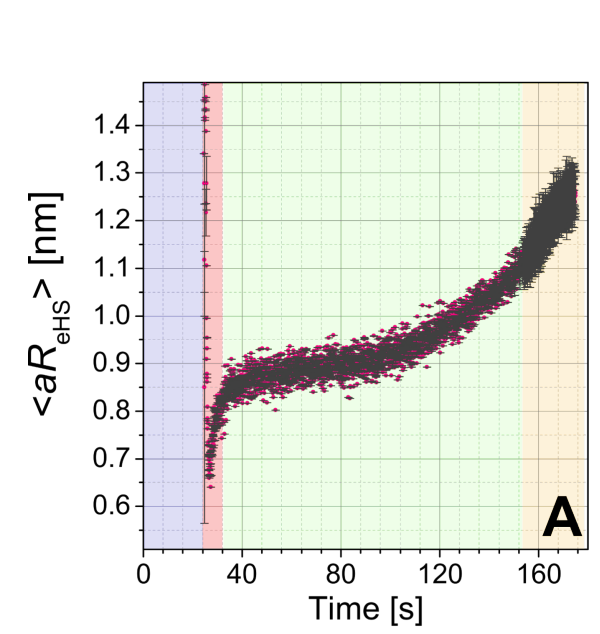

\section{Period: | ||}

III
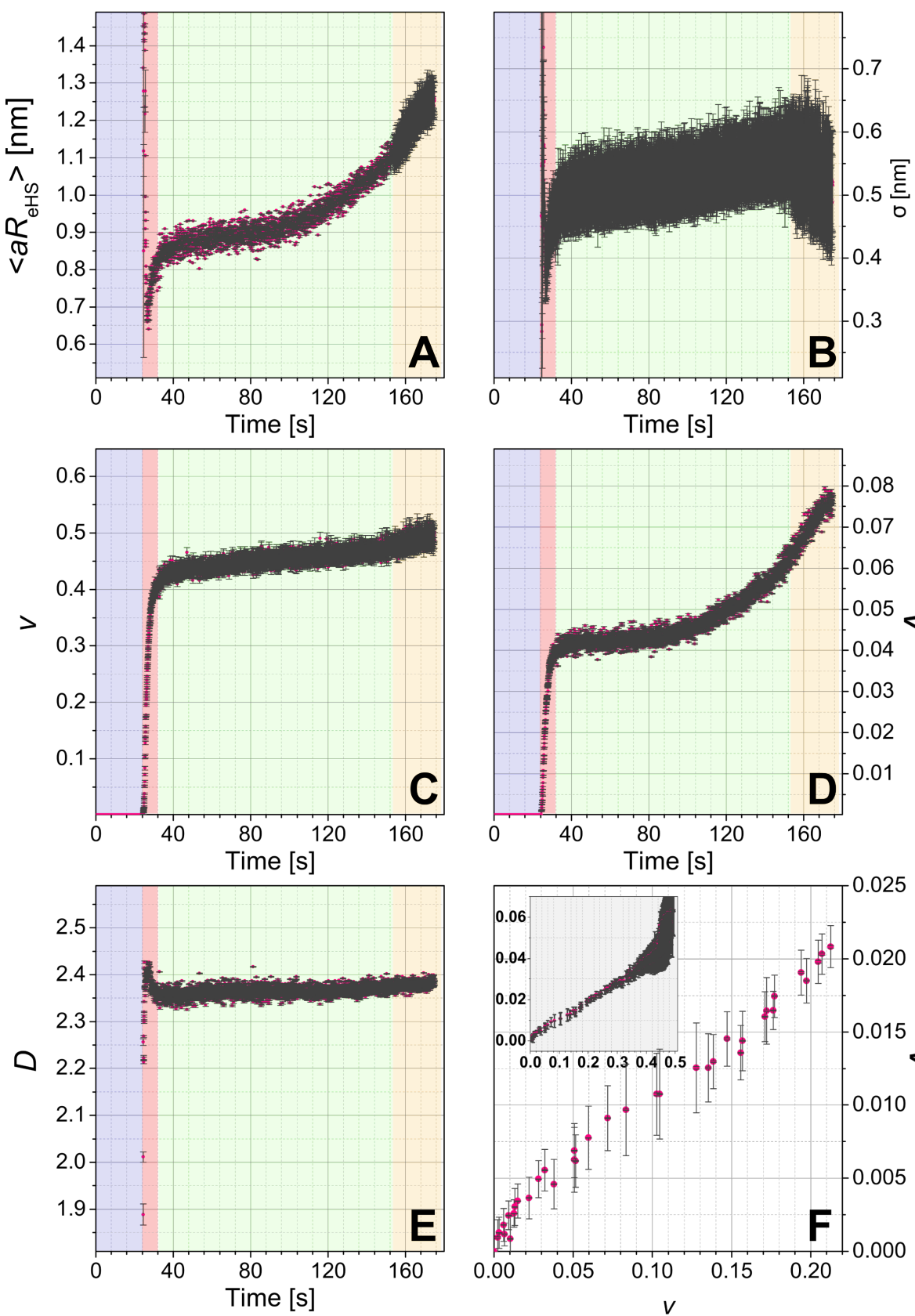
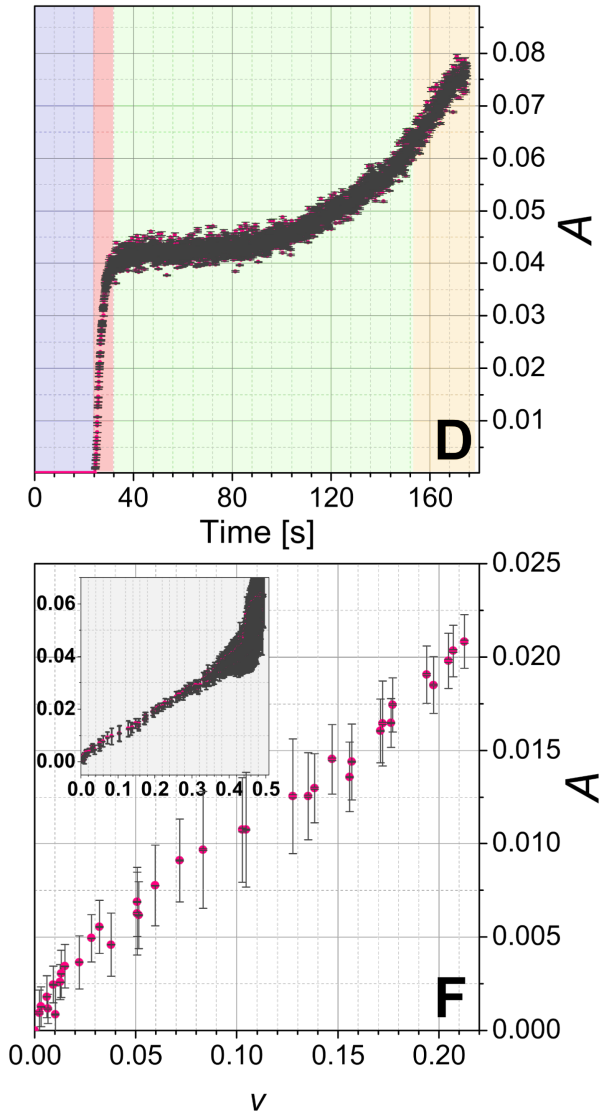

Figure 4: Evolution in the crucial parameters of the fitting model plotted as a function of the time. A) Mean additional hard sphere radius $<a R_{\mathrm{eHS}}>$; $\mathrm{B}$ ) the corresponding standard deviation $\sigma ; \mathrm{C}$ ) local volume fraction $\mathrm{v}$; parameters characterising $\left\langle S_{\text {agg }}(q)>\right.$ : $\left.\mathrm{D}\right) A$, relative product of the number density of the aggregates $N_{\mathrm{agg}}$ and their specific surface area $\mathrm{SAA}_{\mathrm{agg}}$; $\mathrm{E}$ ) dimensionality of the aggregate $D ; \mathrm{F}$ ) correlation $A$ vs $\mathrm{v}$, with inset showing the same as the main Figure 4F, but for a wider range; axes legends in the inset correspond to those in the main figure. 
negligible with respect to $S_{\text {agg }}(q)$. As $v$ reaches ca. 0.1 after about $30 \mathrm{~s}$ (transition between regions II and III in Figure 1) the actual evolution of $\left\langle a R_{\mathrm{eHS}}>\right.$ and $\sigma$ begin: $<a R_{\mathrm{eHS}}>$ starts at a value of ca. $0.7 \mathrm{~nm}$ and rapidly increases to ca. $0.9 \mathrm{~nm}$ after $50 \mathrm{~s}$ and then more gradually to ca. $1.1 \mathrm{~nm}$ after $150 \mathrm{~s}$ (period III in Figure 1). The associated standard deviation $\sigma$ follows a similar trend as $<a R_{\mathrm{eHS}}>$, growing from 0.35 to $0.5 \mathrm{~nm}$ after $50 \mathrm{~s}$, and then levels off at ca. $0.5 \mathrm{~nm}$ after $150 \mathrm{~s}$ (end of period III in Figure 1A) within the fitting uncertainty. These initial rapid changes after up to $50 \mathrm{~s}$ are also reflected in the evolution of parameter $v$ (Figure $4 \mathrm{C}$ ), where the parameter rapidly increases to ca. 0.45 and then remains constant within the fitting uncertainty after up to $150 \mathrm{~s}$. Because $<a R_{\mathrm{eHS}}>$ directly relates to the size of lysozyme within the silica aggregate, we can link the changes in $<a R_{\mathrm{eHS}}>$ to possible changes in the structure/folding/shape of the protein. Between ca. 30 and ca. $50 \mathrm{~s}$, where the LZM molecules rapidly induce the aggregation of the silica NPs, the LZM molecules appear to undergo a deformation (compression). However, as this process approaches equilibrium, through the internal densification of the aggregates, the molecules gradually return to their native dimensions. This result is in agreement with findings concerning the activity of lysozyme within composites with silica, showing that smaller silica NPs (as those used for our experiments) promote higher enzymatic activity of lysozyme, and that this, in turn, depends on the preservation of the native shape of the molecule upon composite formation [22].

Furthermore, the formation of the silica-LZM composite is dominated, from the very moment of mixing for the initial $20 \mathrm{~s}$, by a rapid, 4.5-fold increase of parameter $A$ (Figure 4D). This is best explained by the increasing number density of the aggregates, $N_{\text {agg }}$, and the associated increasing specific surface area, $\mathrm{SSA}_{\text {agg }}$ (see Equation 17 and Equation 29 in the Appendix). After $t=40 \mathrm{~s}$, parameter $A$ further increased, albeit at a slower rate. The concurrent evolution of the fractal dimension (parameter $D$; Figure 4E) suggests that initially (up to $50 \mathrm{~s}$ ), the aggregates have a relatively open morphology with $D<2.4$ and characterized by a limited contribution of $<S_{\mathrm{SHS}}(q)>$ due to $v<0.1$ (Figure 4C). Afterwards (after more than $50 \mathrm{~s}$ ), the aggregates reached an internally denser state, as reflected by the steadying of both values for $D$ (ca. 2.4) and $v$ (ca. 0.45). In other words, since these two parameters, $D$ and $v$, reflect the internal structure of the aggregates from the perspective of the two structurefactor contributions (Equation 17), their evolution clearly indicates no further internal changes in the aggregates between 50 and $150 \mathrm{~s}$. If such an internal densification processes had occurred, one would expect that it would have contributed to the decrease of the specific surface area of the aggregates, $\mathrm{SSA}_{\text {agg. }}$. Interestingly, however, parameter $A$ (Figure 4D) keeps increasing after $50 \mathrm{~s}$, i.e., after the internal dense structure is established, meaning that the product of number density of the aggregates and their specific surface area actually increased. This is possibly a result of secondary processes involving the "breakup" of larger aggregates into smaller units. Indeed, if we correlate the changes in $A$ and $v$ (Figure 4F), we observe three stages of such secondary processes. In the first stage for $v<0.1$ (up to $25.5 \mathrm{~s}$ ), $A$ grows as a function of $v$ in a bound exponential mode, which translates into an increasing number of lowdimensional aggregates with hardly any internal correlations, forming an extended network of particles of low dimensionality $D$ (Figure $4 \mathrm{E}$ ). In the second stage, as $v$ increases from 0.1 to ca. 0.45 (25.5 to $70 \mathrm{~s}$ ), $A$ as a function of $v$ (Figure $4 \mathrm{~F}$ ) shows a linear dependence, indicating that as the number density of aggregates increases they also gradually densify, and that the growth of the aggregates occurs at the same rate as their internal densification. Finally, in the third stage, once $v$ remains relatively constant at ca. 0.45 (after $70 \mathrm{~s}$ ), the product of the number density and the specific surface area of the aggregates continues to increase as documented by the increasing value of $A$, yet without any further dramatic changes to the internal structure/arrangement (i.e., constant values of $D$ and $v$ ), implying the aforementioned breakup of the larger aggregates into smaller units. These processes can be best explained as the initial rapid flocculation/clumping of NPs and LZM together into an extensive network just after mixing as the system is out of equilibrium, followed by the gradual evolution towards a steady state, in which smaller aggregates are more favourable.

During period IV (after more than $150 \mathrm{~s}$ ), the time evolution of the three parameters $\left(<a R_{\mathrm{eHS}}\right\rangle, v$ and $\sigma$; Figure $\left.4 \mathrm{~A}-\mathrm{C}\right)$ exhibited a characteristic discontinuity from the trends observed during periods II and III. This is because at times greater than $150 \mathrm{~s}$, the scattering intensity at high $q$ (which corresponds to the form factor, Figure 1D) changed significantly, so that the original form factor of pre-mixing silica NPs from Figure 1B was not representative for silica particles after $150 \mathrm{~s}$. Thus, we could no longer use the fitted size distribution in our model, and any trends of these three parameters (Figure 4A-C) were not valid any more after $150 \mathrm{~s}$. On the other hand, due to the fact that the low- $q$ part of the data by definition is practically independent from the form factor, in fact the evolution of parameters $A$ and $D$ (trends in Figure 4D,E), even after $150 \mathrm{~s}$ are representative for the processes at the length scales corresponding to entire aggregates. However, due to the fact that in period IV our scattering model is no longer self-consistent, we did not analyse those trends.

\section{Implications}

The analysis of the evolution of the fitting parameters (Figure 4) draws a clear image of the four-step sequence of events during 
aggregation induced by the interaction between the protein LZM and amorphous silica NPs (Figure 5).

Immediately upon mixing, aggregation is induced due to the opposing surface charge of the silica NPs and the protein. An infinitely extensive and open ( $D=1.8-2.2)$ aggregate network, from the point of view of the SAXS measurement, forms within ca. four data frames (ca. $200 \mathrm{~ms}$ ). The so-formed network initially has no internal correlations, as is expected for a classical mass fractal [46]. However soon after (ca. $1 \mathrm{~s}$ ), areas of correlated NPs-LZM domains start forming within the network and the increase in the internal volume fraction, $v$, indicates an internal densification and ordering. This is also reflected by the fact that parameter $D$ reaches a stable and relatively high value of ca. 2.4, which is characteristic for denser mass fractals. Such a fractal dimension for silica-lysozyme aggregates was previously reported $[16,23]$ and can be associated with the diffusionlimited particle-cluster aggregation (DLPCA) mechanism [4752]. The DLPCA growth mode is also evidenced not only by the plateau value of $D$, but also through the fact that the value of $D$ increases with the size/extent of the aggregates [47,49] expressed indirectly by the parameter $A$ (see region II in Figure 4D-E). This means that aggregates grow through the accretion of individual primary particles to larger aggregates [52], where aggregates as such become denser as their size in- creases, which in consequence favours the eventual occurrence of the correlated domains (which are the ultimate dense regions in the aggregate composed of smaller particles).

The parameters characterizing the interparticle correlation effects carry information about the size of the LZM molecules bridging the silica NPs. From Figure 4A-B it is clear that in this network the dimensions of the protein molecules are considerably smaller than the native dimensions of lysozyme in any possible orientation. Hence, this suggests that initially the binding of silica NPs by lysozyme involves a severe deformation/unfolding of the protein molecules, followed in time by a relaxation and increase in the protein dimensions towards a (more) native state (Figure 5). The final value of the radius of $1.1 \pm 0.5 \mathrm{~nm}$ for the protein, which is reached before $150 \mathrm{~s}$, is close to a radius of the protein in a side-on orientation (ca. $1.5 \mathrm{~nm}$ ), rather than in the end-on orientation (ca. $2.25 \mathrm{~nm}$ ). Our ex situ analysis of the dried composite samples (see Experimental section) showed that at $1000 \mathrm{ppm}$ lysozyme, $32.7 \mathrm{wt} \%$ of the protein was incorporated into the composites. This means that for silica NPs precipitated at the concentration of $0.8737 \mathrm{~g} / \mathrm{L}$ (calculated from the volume distribution in the SAXS patterns), the concentration of lysozyme in the composite was $0.4245 \mathrm{~g} / \mathrm{L}$. This is valid under the assumption that all available silica NPs were bound in aggregates with lysozyme.

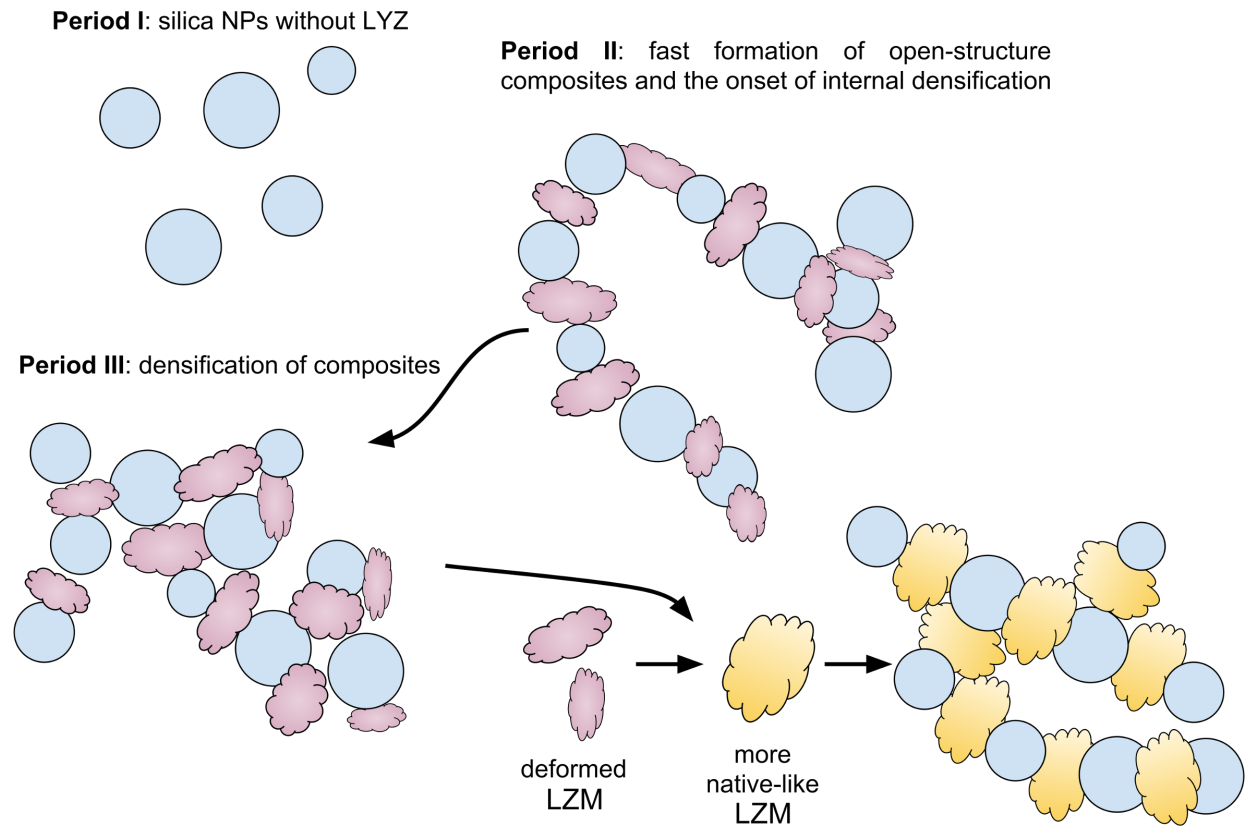

Figure 5: Schematic of the selected steps in the formation of silica-LZM composites as derived from the fast in situ and time-resolved SAXS data. Period I - unaggregated silica NPs (spherical in shape blue objects) before LZM addition. Following the LZM addition (cloud-like purple and yellow objects) occurs period II - fast aggregation to low-dimensionality fractal networks constituting the internal structure of large composite particles (primarily $\mathrm{S}_{\text {agg }}(q)$ contribution); period III - internal densification of the network in the process of which the interparticle correlations develop (evolution of $<S_{\mathrm{SHS}}(q)>$ and $S_{\mathrm{agg}}(q)$ ). At the early stages of densification the lysozyme molecules are strongly deformed within the aggregates; during further densification the protein molecules appear to relax to a more native structure. 
Hence, the number density of the protein molecules was $N_{\mathrm{LZM}} \approx 1.8 \cdot 10^{19} \mathrm{~L}^{-1}$, and for silica $N_{\mathrm{NP}} \approx 1.8 \cdot 10^{19} \mathrm{~L}^{-1}$ (from SAXS by converting the volume distribution to a number distribution). This directly suggests that the silica-lysozyme aggregates are near almost stoichiometric, with one protein molecule associated with one silica NP. Such a stoichiometric relationship is actually expected for small silica particles of the size close to that of the protein molecule [22,53]. Su et al. [54] found that at small surface coverage the lysozyme attaches to silica NPs in a side on orientation, and recently the molecular dynamics simulations by Hildebrand et al. [55] also further confirmed that the side-on orientation of lysozyme with respect to silica constitutes the configuration of the highest attraction. This together with the relatively low dipole moment and the positive surface charge of the protein surface, potentially accounts for the bridging of the NPs by the LZM molecules, as the protein does not show a favoured orientation of the opposite active sites in the side-on orientation (i.e., both active sites show similar binding properties). In such a case one should indeed expect the DLPCA mode of aggregation, with the binding of the protein to the silica NP surfaces taking place through specific amino acids at the opposite sides of the molecule [24,55-57]. The densification of the internal structure of the aggregates reaches a steady point, when the LZM molecules relax to their native-like dimensions. Yet, at the same time the actual network constituting the composite, appears to break up into smaller aggregate units. The morphological changes of the composites further continue beyond $150 \mathrm{~s}$. This is documented through the change in the form factor of the silica NPs that appear to grow in size, compared to the pre-mixed initial NPs. Although we cannot use our model to explain this last stage, we can speculate that the observed change is caused by a partial coalescence or Ostwald ripening of NPs inside of the aggregates. It is well documented that silica NPs synthesized from monosilicic acid are internally highly disordered and hydrated at their surfaces [31]. Previous studies found that the initially formed NPs if aggregated continued their growth, resulting in some cases in larger homogeneous silica spheres $[31,58,59]$, and this may explain why silica is such a persistent scaling material in hydrothermal systems.

\section{Conclusion}

The analysis of in situ scattering data collected at 20 fps from the formation of silica-lysozyme composites showed that the processes included the formation of large aggregated structures in which individual silica NPs were bridged by LZM molecules. We developed and applied a new scattering model to underpin the changes of the morphology of the composites as a function of time. This model allowed us to unravel that the formation follows a diffusion limited particle-cluster aggregation (DLPCA) mechanism, which results in relatively densely packed mass-fractal-like aggregates within which non-fractal correlated domains of particles evolve. Furthermore, we used the scattering model to link the evolution in the measured structure factor to the lysozyme molecule, and we found that the aggregation processes involve severe deformation of the protein molecules, which is then followed by the relaxation towards the original dimensions.

\section{Appendix \\ Derivation of Equation 17}

The contribution of $S_{\text {int }}(q)$ in Equation 17 has a rather simple form. However, the actual meaning of the parameter $A$ extends beyond this short representation. Below, we present the derivation of the equation and we indicate the approximations we make on the way to the final form of the equation.

First we consider a structure factor function describing an arrangement of primary particles of a certain scattering length density (SLD), $\rho_{\mathrm{p}}$, within an aggregate. The SLD of the space between the particles within the aggregate (the "template") is $\rho_{t}$, while the SLD of the surrounding ("solvent") is $\rho_{\mathrm{s}}$. The average scattering contrast of the aggregate, $\Delta \rho_{\text {agg }}$, is then:

$$
\Delta \rho_{\mathrm{agg}}=v\left(\rho_{\mathrm{p}}-\rho_{\mathrm{s}}\right)+(1-v)\left(\rho_{\mathrm{t}}-\rho_{\mathrm{s}}\right)
$$

where $v$ is a local volume fraction of particles within the aggregate (as in Equation 5). We also define any contrast fluctuation within an aggregate as:

$$
\Delta \eta=\rho_{\mathrm{p}}-\rho_{\mathrm{t}}
$$

If we write the structure function of an aggregate ("template") as $S_{\text {agg }}(q)$ and the internal arrangement within this aggregate as $S_{\text {int }}(q)$, then similar to Hashimoto et al. [43] and Lin et al. [44], we can express the scattering intensity by the generalized Equation 22 , in which “ $\otimes$ " denotes a convolution operation of the functions:

$$
\begin{aligned}
S_{\mathrm{eff}}(q) & =S_{\mathrm{agg}}(q)+\frac{V_{\mathrm{p}} \cdot \phi_{\mathrm{p}} \cdot \Delta \eta^{2}}{V_{\mathrm{agg}} \cdot \phi_{\mathrm{agg}} \cdot \Delta \rho_{\mathrm{agg}}^{2}} S_{\mathrm{agg}}(q) \otimes S_{\mathrm{int}}(q) \\
& \approx S_{\mathrm{agg}}(q)+\frac{V_{\mathrm{p}} \cdot \phi_{\mathrm{p}} \cdot \Delta \eta^{2}}{V_{\mathrm{agg}} \cdot \phi_{\mathrm{agg}} \cdot \Delta \rho_{\mathrm{agg}}^{2}} S_{\mathrm{int}}(q),
\end{aligned}
$$

where $V_{\text {agg }}$ and $\phi_{\text {agg }}$ represent the volume and the volume fraction of aggregates in the solution, respectively. Here, $S_{\text {int }}(q)=S_{\mathrm{SHS}}(q)$ from Equations 5-14, whereas the $S_{\text {agg }}(q)$ contributes to the increase in intensity at low $q$ in the course of the 
formation of aggregates. The approximation in Equation 22 is valid when the overall radii of the aggregates, $\xi$, are significantly larger that the interparticle correlation distance from Equations 5-14 ( $\left.\xi>>R_{\mathrm{HS}}\right)$. Furthermore, we must re-normalize $S_{\text {eff }}(q)$ in a different way than Hashimoto et al. and Lin et al. in order to combine it with the form factor defined in Equation 1 as for our scattering data the intensity for $q \rightarrow 0$ in a $\log -\log$ representation does not level off to a finite value (Figure 2 and Figure 3). Consequently, it is impossible to determine $V_{\text {agg. }}$. On the other hand the radii of the primary particles and, consequently, their volume, $V_{\mathrm{p}}$, are known, and therefore it is reasonable to normalize $\mathrm{S}_{\mathrm{eff}}(q)$ with respect to the primary particle volume rather than the aggregate volume:

$$
S_{\mathrm{eff}}(q)=S_{\mathrm{int}}(q)+\frac{V_{\mathrm{agg}} \cdot \phi_{\mathrm{agg}} \cdot \Delta \rho_{\mathrm{agg}}^{2}}{V_{\mathrm{p}} \cdot \phi_{\mathrm{p}} \cdot \Delta \eta^{2}} S_{\mathrm{agg}}(q)
$$

Now let us assume that the aggregates follow mass-fractal behaviour and that the correlation function of mass fractals is described $[60,61]$ by Equation 24 :

$$
g_{\text {agg }}(r)=K \cdot r^{D-d} \text {, }
$$

where $D$ is a fractal dimension, $d$ is the Euclidean dimension $(d=3)$ and $K$ is a normalization constant that is proportional to the mass and surface area of an aggregate. We do not include a cut-off function since in our case the intensity did not level off at low $q$ as mentioned above. For the sake of simplicity let us assume again that the electron density of the aggregates is homogeneously distributed and its corresponding correlation function only involves a two-phase system. In this case the specific surface area of aggregates $\left(\mathrm{SSA}_{\mathrm{agg}}\right)$ is proportional to the derivative of the correlation function at the near-zero length scale $r \rightarrow 0$ :

$$
\mathrm{SSA}_{\mathrm{agg}}=-4 \cdot \phi_{\mathrm{agg}}\left(1-\phi_{\mathrm{agg}}\right) \cdot \lim _{r \rightarrow 0} \frac{\mathrm{d} g_{\mathrm{agg}}(r)}{\mathrm{d} r}
$$

This is valid for the considered small volume fractions $\left(1-\phi_{\text {agg }} \approx 1\right)$. The limit in Equation 25 exists essentially only for $D=4$, and since $D<4, \mathrm{SSA}_{\text {agg }}$ becomes increasingly larger for decreasing length scales, yielding an infinite surface area at an infinitely small length scale $r$. However, since the aggregates are composed of primary particles with a typical radius, $R_{\mathrm{HS}}$, we can say that the aggregate does not contain smaller features than those primary particles (i.e., $r \geq R_{\mathrm{HS}}$ ). Therefore, we find a finite specific surface area for mass fractal aggregates for $\lim \left(r \rightarrow R_{\mathrm{HS}}\right)$

$$
\begin{aligned}
\mathrm{SSA}_{\mathrm{agg}} & =4 \cdot(3-D) \cdot \phi_{\mathrm{agg}} \cdot K \cdot R_{\mathrm{HS}}^{D-4} \\
& \Rightarrow K=\frac{\mathrm{SSA}_{\mathrm{agg}} \cdot R_{\mathrm{HS}}^{4-D}}{4 \cdot(3-D) \cdot \phi_{\mathrm{agg}}} .
\end{aligned}
$$

This way this newly derived $K$ constant in Equation 26 substitutes the $K$ constant from Equation 24 and thus, we can use the correlation function from Equation 24 to calculate the structure factor. Please note the Hashimoto et al. described the structure factor in such a way that it is normalized as a form factor, i.e., it is normalized by the total volume. This is in line with the structure factor of mass-fractal aggregates as described by Sorensen and Wang [60], yet it is different from a better-known derivation by Teixeira [62]. Both Sorensen and Wang's and Teixeira's approaches are valid as long as one considers normalizations explicitly. The structure factor is described by the rotation-averaged Fourier transform:

$$
\begin{aligned}
S_{\mathrm{agg}}(q)= & \frac{N_{\mathrm{agg}}}{V_{\mathrm{agg}}} \int_{0}^{\infty} g_{\mathrm{agg}}(r) \cdot 4 \pi r^{2} \frac{\sin (q r)}{q r} \mathrm{~d} r \\
= & \frac{\pi \cdot \Gamma(D-1) \cdot N_{\mathrm{agg}} \cdot \mathrm{SSA}_{\mathrm{agg}} \cdot R_{\mathrm{HS}}^{4-D}}{V_{\mathrm{agg}} \cdot \phi_{\mathrm{agg}} \cdot(3-D)} \\
& \cdot \sin \left(\frac{(D-1) \pi}{2}\right) \cdot q^{-D},
\end{aligned}
$$

where $N_{\text {agg }}$ is the number density of aggregates and $\mathrm{SSA}_{\text {agg }}$ is their specific surface area. By substitution of Equation 27 into Equation 23, we obtain:

$$
\begin{aligned}
S_{\mathrm{eff}}(q)= & S_{\mathrm{int}}(q)+\frac{\Delta \rho_{\mathrm{agg}}^{2}}{\Delta \eta^{2}} \cdot \frac{\pi \cdot \Gamma(D-1)}{(3-D)} \\
& \cdot \frac{N_{\mathrm{agg}} \cdot \mathrm{SSA}_{\mathrm{agg}} \cdot R_{\mathrm{HS}}^{4-D}}{V_{\mathrm{p}} \cdot \phi_{\mathrm{p}}} \cdot \sin \left(\frac{(D-1) \pi}{2}\right) \cdot q^{-D}
\end{aligned}
$$

For spherical primary particles $V_{\mathrm{p}}$ is known and a final form of the equation for the effective structure factor could be derived:

$$
\begin{aligned}
S_{\text {eff }}(q) & =S_{\text {int }}(q)+\frac{\Delta \rho_{\mathrm{agg}}^{2}}{\Delta \eta^{2}} \cdot \frac{\Gamma(D-1)}{(3-D)} \cdot \frac{3 N_{\mathrm{agg}} \cdot \mathrm{SSA}_{\mathrm{agg}}}{4 \phi_{\mathrm{p}} \cdot R_{\mathrm{HS}}^{D-1}} \\
& \cdot \sin \left(\frac{(D-1) \pi}{2}\right) \cdot q^{-D} \\
& =S_{\text {int }}(q)+A \cdot q^{-D}
\end{aligned}
$$

In Equation 29 we introduced several simplifications. Firstly, $N_{\text {agg }}, \mathrm{SSA}_{\text {agg }}, \Delta \eta$ and $\Delta \rho_{\text {agg }}$ are essentially unknown, and it is impossible to determine any one of these parameters independently. They have to be combined into a collective parameter. 
This is necessary as Equation 29 was derived for a system characterized by a monodisperse particle distribution with only a single value of $\phi_{\mathrm{p}}$ for a given $R_{\mathrm{HS}}$ and the resulting $N_{\mathrm{agg}}$. For a polydisperse distribution, as in our study, although a population of primary particles is described by $D\left(r_{i}\right)$ (Equation 2), the resulting distribution of aggregate sizes will be totally independent from this initial distribution, and it will also be unpredictable. Secondly, the $R_{\mathrm{HS}}{ }^{1-D}$ component in Equation 29 could be important, since it determines the high- $q$ cut-off at which the contribution of the structure to the intensity lessens, and the form factor dominates. In Equation 29 this very transition point is dominated by a $S_{\text {int }}(q)$ contribution and its strong correlation peak. Hence, we assume that $R_{\mathrm{HS}}{ }^{1-D} \approx 1$. Thirdly, the remaining part of the expression depending on parameter $D$ is practically constant at a value of ca. 1.2, and although we could introduce it explicitly in the model it does not affect the final trends. Hence, as a result of the above approximations, we use $A$ as a single collective fitting parameter in our model. Changes in $A$ therefore should be interpreted primarily as the average change of the product of the number density of aggregates and their specific surface area, and these two physical parameters are related to the size (or "extent") of the aggregates.

\section{Experimental \\ Synthesis of amorphous silica-lysozyme composites}

Sodium metasilicate $\left(\mathrm{Na}_{2} \mathrm{SiO}_{3} \cdot 5 \mathrm{H}_{2} \mathrm{O}\right.$, technical grade), hen eggwhite lysozyme (crystalline, powdered, $>90 \%$ pure, residual sodium acetate and chloride) and $\mathrm{HCl}(37 \%$, analytical grade) were purchased from Sigma-Aldrich. Separate stock solutions of dissolved silica $\left(\mathrm{SiO}_{2}=1000 \mathrm{ppm}, \mathrm{pH} 12.5\right)$ and lysozyme (5 wt \%, pH 3.5) were prepared by dissolving the required amount of sodium metasilicate or lysozyme in ultrapure deionised water $(18.2 \mathrm{M} \Omega \cdot \mathrm{cm})$. Silica NPs were prepared in a $500 \mathrm{~mL}$ plastic reactor by neutralizing the silica stock solution through titration with $\mathrm{HCl}$ until $\mathrm{pH} 7.5$ was reached. This neutralized solution was left to polymerize and age for $16 \mathrm{~h}$. Silica-protein composites were obtained by mixing the silica NP solution with a pre-measured amount of the lysozyme stock solution under rapid stirring $(500 \mathrm{rpm})$ to yield a $\mathrm{SiO}_{2} \mathrm{NPs}$ solution with $1000 \mathrm{ppm}$ lysozyme (final pH 6.9, salinity $20 \mathrm{mM}$ ).

\section{Scattering experiments}

The formation process and the development of the structure of the silica-LZM composites was studied in situ and in a timeresolved manner by using synchrotron-based small-angle X-ray scattering (SAXS) at the BioSAXS beamline [63] P12 of the EMBL at PETRA III (DESY, Germany) using a monochromatic X-ray beam at $10 \mathrm{keV}$. Two-dimensional scattered intensities were collected at small angles with a Dectris Pilatus $2 \mathrm{M}$
(2D large-area pixel array detector) using an acquisition time of $50 \mathrm{~ms}$ per frame. Transmission was measured by means of a photodiode installed in the beam stop of the SAXS detector. A sample-to-detector distance of ca. $3 \mathrm{~m}$ allowed for a usable $q$-range of $0.04<q<4.5 \mathrm{~nm}^{-1}$. The scattering-range at small angles was calibrated against silver behenate, and the intensity was calibrated to absolute units against water. For the in situ experiment, first, the starting silica NP solution was continuously circulated between the reactor (where the suspension was stirred at $500 \mathrm{rpm}$ ) and the flow-through cell with embedded quartz capillary (ID $1.7 \mathrm{~mm}$, wall thickness $50 \mu \mathrm{m}$; aligned with the X-ray beam) using a peristaltic pump (Gilson MiniPuls 3, flow ca. $500 \mathrm{~mL} / \mathrm{min}$; tubing: ID $2 \mathrm{~mm}$, total length $2 \mathrm{~m}$; reactor-tocell-distance: $0.7 \mathrm{~m}$ of tubing). All experiments were conducted at $21{ }^{\circ} \mathrm{C}$. Once a SAXS baseline for the silica NP solution was recorded, the pre-measured amount of lysozyme stock solution was pumped into the reactor at a fast rate. This injection was done remotely from the operator hutch via a $10 \mathrm{~m}$ long PTFE tube (ID $4 \mathrm{~mm}$ ) that was routed into the reactor located in the experimental hutch. The tube was filled in such a way that the LZM solution was located in the last ca. $40 \mathrm{~cm}$ of the tube on the reactor side. The other end of the tube in the operator room was equipped with a $50 \mathrm{~mL}$ syringe filled with air. Thus the experiment started with recording of $24 \mathrm{~s}(480 \times 50 \mathrm{~ms})$ SAXS patterns of the silica NP solution circulating through the capillary, prior to the fast injection of the entire content of the tube containing the lysozyme with a single rapid push of the syringe plunger that lasted ca. 200-400 ms. This fast injection rate in combination with the fast stirring in the reactor (500 rpm), pumping $(500 \mathrm{~mL} / \mathrm{min})$ and fast data acquisition (50 ms per SAXS pattern) provided the best possible conditions for the characterisation of all the steps leading to the formation of the silica-LZM composites. The used experimental set up introduced an unavoidable dead-time of ca. $500 \mathrm{~ms}$ between the injection moment and the first actual measurement of the mixed solution, i.e., the time required for the mixed solution to reach the capillary where the SAXS pattern was recorded. In order to be able to analyse and model the silica-LZM composite scattering patterns we also acquired a series of backgrounds and reference samples including an empty capillary and a capillary filled with water, silica stock solution, LZM solutions at different concentrations. The initial SAXS data processing and reduction included a series of automatic post-data-collection steps including masking of undesired pixels, normalizations and correction for transmission, instrumental background subtraction and data integration of the collected 2D data to 1D. Further data processing and water background subtraction, model fitting, validation and analysis, were performed through a custom-made script developed in GNU Octave [64,65]. The script we developed as well as all the documentation and the selected scattering curves are available at [45]: https://github.com/ 
tomaszstawski/SilicaLysozymeSAXS. In a first instance for the model, we obtained the size distribution of the initial silica NPs from a Monte Carlo fitting implemented [29,30] in MCSAS under the assumption that the silica NPs particles were spherical [31].

\section{Characterization of dry samples}

To cross-correlate the in situ SAXS data, the silica-lysozyme suspensions were dried in an oven at $40{ }^{\circ} \mathrm{C}$ for ca. $48 \mathrm{~h}$. The resulting powders were washed five times with MilliQ water to remove excess lysozyme and salts followed by a 2 nd drying step at $40{ }^{\circ} \mathrm{C}$. The amount of lysozyme associated with the composites was quantified by determining the total carbon content in solids by mass spectrometry (DELTAplusXL ThermoFisher) with a Carlo-Erba NC2500. From these analyses the lysozyme content was calculated using the molecular formula $\mathrm{C}_{613} \mathrm{H}_{959} \mathrm{~N}_{193} \mathrm{O}_{185} \mathrm{~S}_{10}$ and the molecular weight of $14313 \mathrm{~g} / \mathrm{mol}$ for lysozyme [66] (ProtParam based on UniProtKB entry P00698).

\section{Supporting Information}

\section{Supporting Information File 1}

Additional experimental data.

[https://www.beilstein-journals.org/bjnano/content/ supplementary/2190-4286-10-17-S1.pdf]

\section{Acknowledgements}

This research was made possible by two Marie Curie grants from the European Commission: the NanoSiAl Individual Fellowship, Project No. 703015 and the MINSC Initial Training Research network, Project No. 290040. We also acknowledge the financial support of the Helmholtz Recruiting Initiative grant No. I-044-16-01 and we thank EMBL Hamburg for granting us beamtime at BioSAXS beamline P12 at the PETRA III storage ring (DESY, Hamburg, Germany).

\section{ORCID ${ }^{\circledR}$ iDs}

Tomasz M. Stawski - https://orcid.org/0000-0002-0881-5808 Liane G. Benning - https://orcid.org/0000-0001-9972-5578

\section{References}

1. Patwardhan, S. V. Chem. Commun. 2011, 47, 7567. doi:10.1039/c0cc05648k

2. Shemetov, A. A.; Nabiev, I.; Sukhanova, A. ACS Nano 2012, 6 , 4585-4602. doi:10.1021/nn300415x

3. Coradin, T.; Lopez, P. J. ChemBioChem 2003, 4, 251-259. doi:10.1002/cbic. 200390044
4. Belton, D. J.; Deschaume, O.; Perry, C. C. FEBS J. 2012, 279 , 1710-1720. doi:10.1111/j.1742-4658.2012.08531.x

5. Franzmann, E.; Khalil, F.; Weidmann, C.; Schröder, M.; Rohnke, M.; Janek, J.; Smarsly, B. M.; Maison, W. Chem. - Eur. J. 2011, 17, 8596-8603. doi:10.1002/chem.201100715

6. Frasca, S.; Richter, C.; von Graberg, T.; Smarsly, B. M.; Wollenberger, U. Eng. Life Sci. 2011, 11, 554-558. doi:10.1002/elsc.201100079

7. Rao, A.; Drechsler, M.; Schiller, S.; Scheffner, M.; Gebauer, D.; Cölfen, H. Adv. Funct. Mater. 2018, 28, 1802063. doi:10.1002/adfm.201802063

8. Rao, A.; Cölfen, H. Mineralization Schemes in the Living World: Mesocrystals. New Perspectives on Mineral Nucleation and Growth; Springer: Berlin, Germany, 2017; pp 155-183. doi:10.1007/978-3-319-45669-0_8

9. Tobler, D. J.; Rodriguez-Blanco, J. D.; Dideriksen, K.; Bovet, N.; Sand, K. K.; Stipp, S. L. S. Adv. Funct. Mater. 2015, 25, 3081-3090. doi:10.1002/adfm.201500400

10. De Yoreo, J. J.; Gilbert, P. U. P. A.; Sommerdijk, N. A. J. M.; Penn, R. L.; Whitelam, S.; Joester, D.; Zhang, H.; Rimer, J. D. Navrotsky, A.; Banfield, J. F.; Wallace, A. F.; Michel, F. M.; Meldrum, F. C.; Colfen, H.; Dove, P. M. Science 2015, 349, aaa6760. doi:10.1126/science.aaa6760

11. Helminger, M.; Wu, B.; Kollmann, T.; Benke, D.; Schwahn, D.; Pipich, V.; Faivre, D.; Zahn, D.; Cölfen, H. Adv. Funct. Mater. 2014, 24 3187-3196. doi:10.1002/adfm.201303547

12. Gebauer, D.; Kellermeier, M.; Gale, J. D.; Bergström, L.; Cölfen, H. Chem. Soc. Rev. 2014, 43, 2348-2371. doi:10.1039/c3cs60451a

13. Cölfen, H.; Antonietti, M. Langmuir 1998, 14, 582-589. doi:10.1021/la970765t

14. Mann, S.; Ozin, G. A. Nature 1996, 382, 313-318. doi:10.1038/382313a0

15. Fratzl, P. J. Stat. Phys. 1994, 77, 125-143. doi:10.1007/bf02186835 16. Kumar, S.; Aswal, V. K.; Callow, P. Langmuir 2014, 30, 1588-1598. doi:10.1021/la403896h

17. Bharti, B.; Meissner, J.; Klapp, S. H. L.; Findenegg, G. H. Soft Matter 2014, 10, 718-728. doi:10.1039/c3sm52401a

18. Cardoso, M. B.; Luckarift, H. R.; Urban, V. S.; O'Neill, H.; Johnson, G. R. Adv. Funct. Mater. 2010, 20, 3031-3038. doi:10.1002/adfm.201000144

19. Luckarift, H. R.; Dickerson, M. B.; Sandhage, K. H.; Spain, J. C. Small 2006, 2, 640-643. doi:10.1002/smll.200500376

20. Parks, G. A. Chem. Rev. 1965, 65, 177-198. doi:10.1021/cr60234a002

21. van den Heuvel, D. B.; Stawski, T. M.; Tobler, D. J.; Wirth, R.; Peacock, C. L.; Benning, L. G. Front. Mater. 2018, 5, 19. doi:10.3389/fmats.2018.00019

22. Vertegel, A. A.; Siegel, R. W.; Dordick, J. S. Langmuir 2004, 20 , 6800-6807. doi:10.1021/la0497200

23. Kumar, S.; Aswal, V. K.; Kohlbrecher, J. Langmuir 2011, 27 10167-10173. doi:10.1021/la201291k

24. Bharti, B.; Meissner, J.; Findenegg, G. H. Langmuir 2011, 27 , 9823-9833. doi:10.1021/la201898v

25. Stawski, T. M.; Benning, L. G. Methods Enzymol. 2013, 532, 95-127. doi:10.1016/b978-0-12-416617-2.00005-9

26. ten Elshof, J. E.; Besselink, R.; Stawski, T. M.; Castricum, H. L. Time-Resolved Small-Angle X-Ray Scattering. The Sol-Gel Handbook; Wiley-VCH Verlag GmbH: Weinheim, Germany, 2015; pp 673-712. doi:10.1002/9783527670819.ch21 
27. Shukla, A.; Mylonas, E.; Di Cola, E.; Finet, S.; Timmins, P.; Narayanan, T.; Svergun, D. I. Proc. Natl. Acad. Sci. U. S. A. 2008, 105 5075-5080. doi:10.1073/pnas.0711928105

28. Blake, C. C. F.; Koenig, D. F.; Mair, G. A.; North, A. C. T.; Phillips, D. C.; Sarma, V. R. Nature 1965, 206, 757-761. doi:10.1038/206757a0

29. Pauw, B. R.; Pedersen, J. S.; Tardif, S.; Takata, M.; Iversen, B. B. J. Appl. Crystallogr. 2013, 46, 365-371. doi:10.1107/s0021889813001295

30. Bressler, I.; Pauw, B. R.; Thünemann, A. F. J. Appl. Crystallogr. 2015, 48, 962-969. doi:10.1107/s1600576715007347

31. Tobler, D. J.; Shaw, S.; Benning, L. G. Geochim. Cosmochim. Acta 2009, 73, 5377-5393. doi:10.1016/j.gca.2009.06.002

32. Parkhurst, D. L.; Appelo, C. A. J. Description of input and examples for PHREEQC version 3: a computer program for speciation, batch-reaction, one-dimensional transport, and inverse geochemical calculations; Techniques and Methods; US Geological Survey, 2013. doi:10.3133/tm6a43

33. Kotlarchyk, M.; Chen, S.-H. J. Chem. Phys. 1983, 79, 2461-2469. doi:10.1063/1.446055

34. Pedersen, J. S. J. Appl. Crystallogr. 1994, 27, 595-608. doi:10.1107/s0021889893013810

35. Baxter, R. J. J. Chem. Phys. 1968, 49, 2770-2774. doi:10.1063/1.1670482

36. Menon, S. V. G.; Manohar, C.; Rao, K. S. J. Chem. Phys. 1991, 95 9186-9190. doi:10.1063/1.461199

37. Sztucki, M.; Narayanan, T.; Belina, G.; Moussaïd, A.; Pignon, F.; Hoekstra, H. Phys. Rev. E 2006, 74, 051504. doi:10.1103/physreve.74.051504

38. Barisik, M.; Atalay, S.; Beskok, A.; Qian, S. J. Phys. Chem. C 2014, 118, 1836-1842. doi:10.1021/jp410536n

39. Hansen, J. P.; McDonald, I. R. Theory of Simple Liquids; Elsevier: Amsterdam, Netherlands, 2006.

40. Baranau, V.; Tallarek, U. Soft Matter 2014, 10, 3826. doi:10.1039/c3sm52959b

41. Farr, R. S.; Groot, R. D. J. Chem. Phys. 2009, 131, 244104. doi:10.1063/1.3276799

42. Al-Raoush, R.; Alsaleh, M. Powder Technol. 2007, 176, 47-55 doi:10.1016/j.powtec.2007.02.007

43. Hashimoto, T.; Tanaka, H.; Koizumi, S.; Naka, K.; Chujo, Y. J. Appl. Crystallogr. 2007, 40 (Suppl. 1), s73-s77. doi:10.1107/s0021889807003445

44. Lin, Y.-C. C.; Chen, C.-Y. Y.; Chen, H.-L. L.; Hashimoto, T.; Chen, S.-A. A.; Li, Y.-C. C. J. Chem. Phys. 2015, 142, 214905. doi:10.1063/1.4921567

45. Stawski, T. M. SilicaLysozymeSAXS. https://github.com/tomaszstawski/SilicaLysozymeSAXS (accessed Aug 8, 2018).

46. Besselink, R.; Stawski, T. M.; Van Driessche, A. E. S.; Benning, L. G. J. Chem. Phys. 2016, 145, 211908. doi:10.1063/1.4960953

47. Mandelbrot, B. B. Phys. A (Amsterdam, Neth.) 1992, 191, 95-107. doi:10.1016/0378-4371(92)90511-n

48. Lin, M. Y.; Lindsay, H. M.; Weitz, D. A.; Klein, R.; Ball, R. C.; Meakin, P. J. Phys.: Condens. Matter 1990, 2, 3093-3113. doi:10.1088/0953-8984/2/13/019

49. Gmachowski, L. Colloids Surf., A 2002, 211, 197-203. doi:10.1016/s0927-7757(02)00278-9

50. Lin, M. Y.; Lindsay, H. M.; Weitz, D. A.; Ball, R. C.; Klein, R.; Meakin, P. Nature 1989, 339, 360-362. doi:10.1038/339360a0
51. Ball, R. C.; Weitz, D. A.; Witten, T. A.; Leyvraz, F. Phys. Rev. Lett. 1987, 58, 274-277. doi:10.1103/physrevlett.58.274

52. Ball, R. C.; Witten, T. A. J. Stat. Phys. 1984, 36, 873-879. doi:10.1007/bf01012946

53. Malmsten, M. Colloids Surf., B 1995, 3, 297-308. doi:10.1016/0927-7765(94)01139-v

54. Su, T. J.; Lu, J. R.; Thomas, R. K.; Cui, Z. F.; Penfold, J. J. Colloid Interface Sci. 1998, 203, 419-429. doi:10.1006/jcis.1998.5545

55. Hildebrand, N.; Köppen, S.; Derr, L.; Li, K.; Koleini, M.; Rezwan, K.; Colombi Ciacchi, L. J. Phys. Chem. C 2015, 119, 7295-7307. doi:10.1021/acs.jpcc.5b00560

56. Kubiak-Ossowska, K.; Mulheran, P. A. Langmuir 2010, 26, 15954-15965. doi:10.1021/la102960m

57. Kubiak-Ossowska, K.; Mulheran, P. A. Langmuir 2010, 26, 7690-7694. doi:10.1021/la101276v

58. Tobler, D. J.; Stefánsson, A.; Benning, L. G. Geobiology 2008, 6 , 481-502. doi:10.1111/j.1472-4669.2008.00179.x

59. van den Heuvel, D. B.; Gunnlaugsson, E.; Gunnarsson, I.; Stawski, T. M.; Peacock, C. L.; Benning, L. G. Geothermics 2018, 76, 231-241. doi:10.1016/j.geothermics.2018.07.006

60. Sorensen, C. M.; Wang, G. M. Phys. Rev. E 1999, 60, 7143-7148. doi:10.1103/physreve.60.7143

61. Sinha, S. K.; Freltoft, T.; Kjems, J. Observation of Power-Law Correlations in Silica-Particle Aggregates by Small Angle Neutron Scattering; Elsevier: Amsterdam, Netherlands, 1984. doi:10.1016/b978-0-444-86912-8.50026-2

62. Teixeira, J. J. Appl. Crystallogr. 1988, 21, 781-785. doi:10.1107/s0021889888000263

63. Blanchet, C. E.; Spilotros, A.; Schwemmer, F.; Graewert, M. A.; Kikhney, A.; Jeffries, C. M.; Franke, D.; Mark, D.; Zengerle, R.; Cipriani, F.; Fiedler, S.; Roessle, M.; Svergun, D. I. J. Appl. Crystallogr. 2015, 48, 431-443. doi:10.1107/s160057671500254x

64. Eaton, J. W. J. Process Control 2012, 22, 1433-1438. doi:10.1016/j.jprocont.2012.04.006

65. Eaton, J. W.; Bateman, D.; Hauberg, S.; Wehbring, R. GNU Octave Version 4.2.1 Manual: A High-Level Interactive Language for Numerical Computations;; 2017.

66. Gasteiger, E.; Hoogland, C.; Gattiker, A.; Duvaud, S.; Wilkins, M. R.; Appel, R. D.; Bairoch, A. Protein Identification and Analysis Tools on the ExPASy Server. The Proteomics Protocols Handbook; Humana Press: Totowa, NJ, U.S.A., 2005; pp 571-607. doi:10.1385/1-59259-890-0:571 


\section{License and Terms}

This is an Open Access article under the terms of the Creative Commons Attribution License (http://creativecommons.org/licenses/by/4.0). Please note that the reuse, redistribution and reproduction in particular requires that the authors and source are credited.

The license is subject to the Beilstein Journal of Nanotechnology terms and conditions: (https://www.beilstein-journals.org/bjnano)

The definitive version of this article is the electronic one which can be found at:

doi:10.3762/bjnano.10.17 